\title{
DE COLEOPTERIS,
}

QUAE

\section{OSCARUS ET ALFREDUS BREHM}

\author{
IN AFRICA LEGERUNT,
}

DISSERUIT

J. H. APETZ.

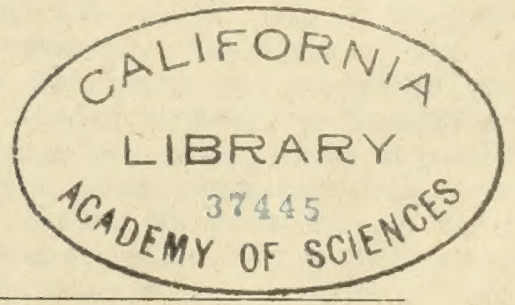

\section{ALTENBURGI,}

EX TYPOGRAPHEO AULIGO.

MDCCCLIV. 


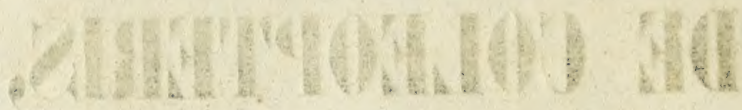

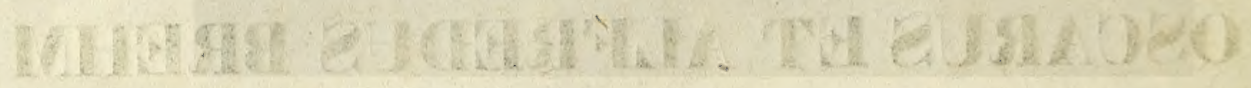

Digitized by the Internet Archive in 2012 with funding from

California Academy of Sciences Library

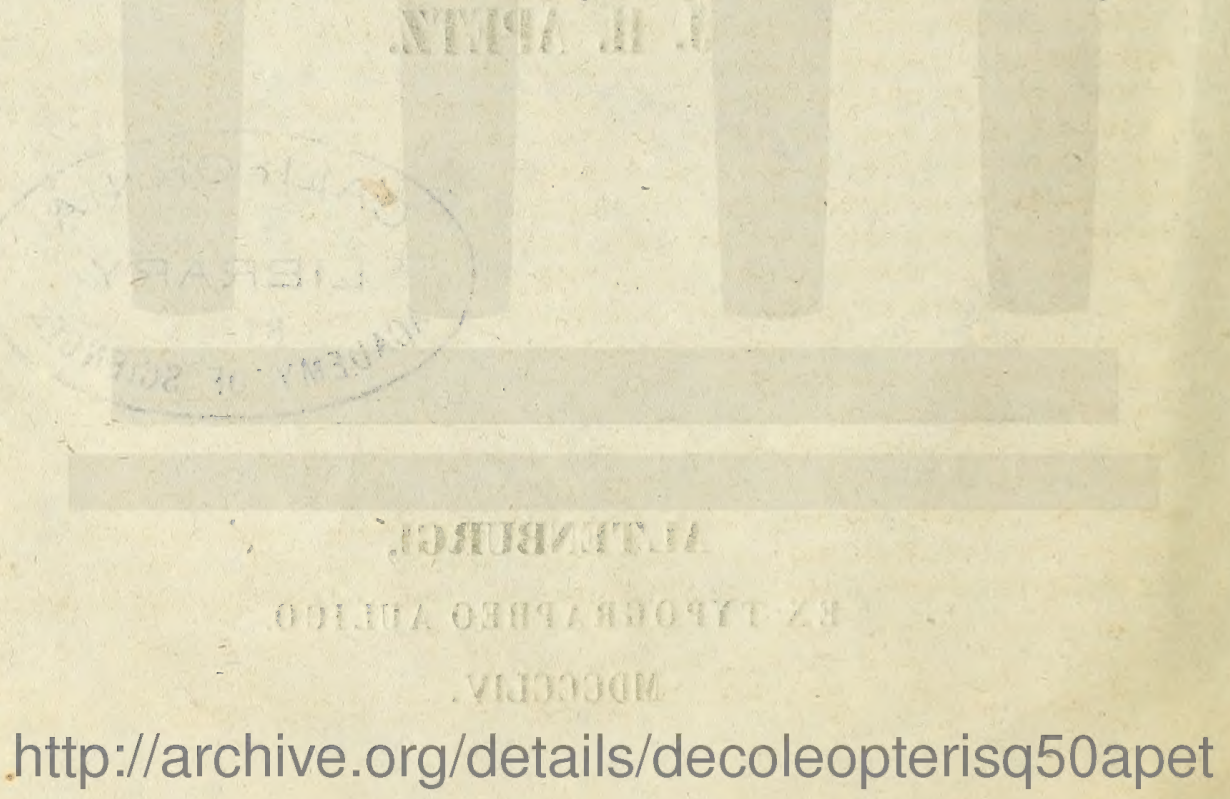


Duae potissimum causae afferri solent, cur geographia insectorum etsi dudum inchoata et a viris doctis adumbrata tamen ne hodie quidem perfecta et omnibus numeris absoluta possit existimari. Primum enim ex iis, qui in hac doctrina explicanda versati sunt, nemo adhuc exstitit, qui tanto ingenii acumine tantaque experientiae copia instructus fuerit, ut, quemadmodum Alexander de Humbold plantarum geographiam, ita insectorum geographiam, ut ita dicam, uno impetu conscriberet et viris harum rerum peritis satisfaceret.

Deinde ei, qui se ad opns tanti laboris accingit, in ipso limine quaestio exoritur, num tempus jamjam adsit, quo sperare liceat fore, ut ejusmodi conatus prospere succedat. Quam viam ingredi oporteat eos, qui in hoc opere studium et operam collocaturi sint, demonstravit Fabricius ${ }^{*}$ ) ille, quem entomologi Linnaeum suum praedicare consueverunt. Vestigia ejus secutus Latreille, plaeclarissimus Gallorum entomologus, qui aedificio ab illo incepto nova eademque firmiora fundamenta substruxit docuitque, quemadmodum in his rebus investigandis versandum esset, accuratius definivit. Omnibus autem, qui postea hoc argumentum tractandum sibi sumserunt, palmam praeripuit Lacordaire ${ }^{* *}$ ). Anno enim 1838 institutionum entomologicarum partem seenndam edidit, eujus in eap. XIV. p. 528 seqq. de geographia insectorum haud scio doctius, an elegantius disseruit. Non equidem vereor, ne viri celeberrimi laudes detrectare videar, quum dico, omnia eum praestitisse, quae tum temporis praestari potuerunt. Ipse enim ingenue profitetur, se dissertationem suam pro adumbratione potius haberi velle, quam pro opere perfecto et absoluto. Se enim reputantem, quam incerta et imperfecta harum rerum cognitione utamur, valde dubitare, num eo loco jam constituti simus, nbi varia haec et disjecta membra in unum corpus colligi et in perfectiorem disciplinae formam redigi possint.

Quae ante tria lustra vera fuerunt, quidni hodie vera esse videantur? Innumerabilia quidem insecta postea in omnibus terrae partibus reperta in indicihns relata sunt. Neque in eo acquiescendum esse putaverunt entomologi, ut eorum formas et partes describerent, colores depingerent, actionum vitalium causas et rationes explicarent; sed rem nimis neglectam retractantes horum animalium stationes et habitationes, quas vocant, diligentius observaverunt, omnia, quae spectant ad situm et

*) Fabricius philosophia entomologica. Hamburgi et Kiliae 1778. p. 154. §. 20.

**) Lacordaire introduction à l'entomologie, T. II. Paris 1838, p. 528. chap. XIV. 
naturam locorum, ubi brevi sua vita defunguntur, accuratius notaverunt et sic materiam cumulaverunt, ex qua qui disciplinae nostrae studium et operam navare cupiat, summam capiet utilitatem.

At hujus apparatus copia desiderio nostro longe inferior est. Quum enim tabulas geographicas perlustramus, animus stupefactus haeret in vastis illis terrae regionibus, quae Europaeorum manibus insectorum rapacibus intactae remanserunt, vel levia tentamma vix perpessae sunt. Longum est, has omnes enumerare et a consilio meo alienum. Advertant, quaeso, lectores animum ad interiorem Africam inter duos Oceanos et circulos tropicos inclusam. Ingens, mehercule, terrarum spatium, cujus in campis et montibus quot insecta vel corporis magnitudine insignia, vel formae insolentia mirabilia, vel colorum varietate et splendore eximia latere suspicari licet, quum Tefflos, Goliathos, Charaxes, alia id genus respicimus! Suspicari licet, inquam; re vera enim totus ille tractus exceptis aliquot regionibus in ora maritima sitis terra incognita est. Nee mirum. Multi Europaei, quos laetissima spe prosequebamur, quum in adyta Africae penetrare conarentur, cum coelo perniciose, cum peregrinationum molestiis, cum infesto incolarum animo misere luctati vel mortem occubuerunt, vel longe a contentionum termino revertere coacti fuerunt, Quo factum est, ut spes omnes, quas de is conceperamus, irritae caderent. Tantum igitur abest, ut a Lacordairei sententia supra commemorata recedentes jam tempus adesse dicamus, qno de ratione, qua insecta supra totum terrarum orbem distributa sint, certius aliquid statui possit, ut vix post longam annorum seriem entomogeographiam (venia sit verbo!) exspectari liceat.

Quae quum ita sint, summas laules merebuntur fortes illi viri, qui neglectis humanioris vitae amoenitatibus nee sudori nec valetudini parcentes, omnia, quae vitam ornant, spernentes, famam sitimque perferentes, multiplici denique mortis periculo circumventi longorum itinerum molestias toleraverunt hoc uno consilio, ut hisforiae naturalis studiis nova subsidia compararent atque auxillia utilissima suppeditarent. In hoc nobilissimo virorum coetu receptos videmus Oscarum et Alfredum Brehm, filios celeberrimi Europae ornithologi, qui avium genera et species summo mentis acumine distinxit earumque mores et amores eo sermonis lepore enarravit, quo nemo unquam alius. Hos utique viros in illorum numerum receptos suspicimus ea cum animi affectione, qua nos commotos sentimus, nbi virtutes et merita in patriae civibus eluxerunt. Recens est famosae expeditionis memoria, quam a Barone de Muller parari accepimus eo consilio, ut ipse cum socïs ad Nili fontes adscenderent, quam longissime meridiem versus progrederentur, ea, quae ad accuratiorem Africae interioris cognitionem pertinerent, omnia diligentissime explorarent. Audivimus de sumptus profusione, suppellectilis magnificentia, rerum necessariarum provisione ita ut nihil praetermisisse videretur expeditionis auctor, quo magnos conatus auspicatis viam patefaceret et praemuniret.

Duci expeditionis, quum socium sibi adjungere cuperet, qui cum caetera animalia, tum maxime aves venandi et conservandi artem ealleret, ab amicis Alfredus Brehmous commendatus est utpote qui studio historiae naturalis a puero deditus et egregia disciplina patris institutus imprimis idoneus esset, qui eum opera sua adjnvaret. Alfredus noster, etsi tune aliam vitae rationem sequeretur, tamen ad altiora conamina evocatus libenter paruit; etenim ah ipso numine divino ad hoc iter 
suscipiendum evocari sibi videbatur. Patria igitur derelicta Tergeste profectus est, unde mare internum secunda navigatione trajectus Alexandriae appulit.

Postea litterae allatae sunt, quae parentum animos de filii sorte sollicitos lenire et ad spes laetissimas erigere possent. At eaedem litterae multo vehementius Oscari fratris animum commoverunt. Imago fratris dilecti in Pharaonum terra nunc avibus rarioribus insidiantis, nunc insecta pulchella captantis, nunc sub umbra palmarum ab excursionis laboribus requiescentis et interdiu et noctu ei ob oculos versabatur et entomologiae amorem aliis negotiis restinctum ad tantum ardorem excitavit, ut ne matris quidem lacrymis retineri posset, quin fratrem sequeretur itineris labores et fructus cum eo partiturus. Oh, quam fatales erant illae lacrymate carissimae matris nune alterum quoque filium sibi ereptum deplorantis!

Ominihus nune minus faustis, quam olim Alfredus, in Africam transjecit; nam post longas demum ambages tempestatibus exagitatus Alexandriam advenit, ubi fratres amplexus et lacrymas miscuerunt. Oscarus navigatione infesta graviter afflictus in morbum incidit. Viribus autem post septimum diem refectis agros Alexandrinos pervagari incepit, ac dum Africae insecta investigabat, cum summa animi delectatione desiderium illud expletnm sensit, quo ex patria in has remotas regiones abreptus erat. Alexandria relicta cum fratre Cahirau profectus est, ubi navigium, quali incolae utuntur, conscenderunt. Adversis Nili undis provecti per Aegyptum et Nubiam in Dongolae provinciam pervenerunt. Oscarus toto itinere, ubicunque temporis et loci opportunitalem nactus est, in terram egressus omnium ordinum insecta corripuit. Quae venationes quum quotidie praedam rariorem praeberent, non jam dubitaverunt, quin, quo longius meridiem versus progrederentur, eo insolentiorem insectorum habitum eoque majorem eorum numerum inventuri essent. Num mirabimur, quod juvenis ardore plenus ingenti novorum usque cognoscendorum ac discendorum cupiditate accendebatur et maximo cum desiderio in Africae interioris areana prospiciebat?

At dies fatalis jam instabat, quo, quam fallaces essent mortalium spes, iterum intellectum est. Oscarus enim d. 8. m. Maji 1850 a fratre rogatus, ut membra diei caloribus laxata Nili aquis refrigeraret, in flumen vix descenderat, quum repente nemine casum graviorem suspicante in undas immersus et de oculorum conspectu subductus est. Frustra alvolavit Alfredus fratri in ultimo vitae discrimine versanti opem laturus. Sed corpus tantnm ejus, quod indigenae ad anxilium ferendum arcessiti post dimidiam demum horam reperire potuerunt, exanimatum in littore depositum est. Neque adstantium planetus, neque fratris lacrymae eum ad vitam revocare potuerunt. Postridie ab expeditionis sociis et proceribus Novac Dongolae oppidi funus solemni pompa elatum in agro christianorum Copticorum sepulcris consecrato conditum est. Quicunque vel domi, vel in funesto itinere cum eo familiariter vixerunt, laudibus extollunt animi ejns candoren, morum prohitatem, sermonum alacritatem, studiorum ardorem et assiduidatem. Quas ingenii virtutes itineris comitibus ita comprobavit, ut praematura ejus morte amico potius, quam socio se orbatos esse profiterentur. Tumulus multis amicorum lacrymis irrigatus locum designat, ubi juvenis deplorandi ossa sepulta jacent. Sit ei terra levis!

Alfredus ex fratris moerore paulum recreatus quo modo iter continuaverit, quemadmodum Chartnmae, Sennatiae oppido desertus fuerit ab iis, qnorum fide fretus 
periculosissimum iter susceperat, quo modo omnium rerum penuria oppressus et infirma corporis valetudine conflictatus animo tamen non defecerit, sed fortiter et tenax propositi provinciam sibi demandatam strenue administraverit, hoc loco non exponam; in libro enim, quem proxime editurus est Alfredus Brehm, haec omnia enarrata iegemus. Illud autem meum esse putavi, ut viro optime merito propter operam, quam assiduam eandemque prosperrimam in rebus entomoligicis posuit, entomologorum nomine gratiam quam maximam in hoc libello testarer. Omne enim tempus, quod ei ex victus cura et ex avium vel venatione vel conservatione relinquebatur, ad insecta omnium ordinum conquirenda adhibuit, idque sibi tanquam pietatis in fratrem officium imposuit, ut scilicet laborem ab illo inceptum persequeretur, nova entomologiae subsidia afferret. Ac maxima sane attulit! Nam in Chartumae agris, ubi diutius commoratus est, et in itineribus, quae ripas Fluminis coerulei legens usque ad $13^{\circ}$ 1. s. extendere potuit, insectorum tum rariorum, tum ad hunc diem incognitorum permagnum numerum congessit. In patriam reversus totam hanc congeriem curis meis demandavit. Quam quum accuratius perlustrarem, facile mihi apparuit, tantam insectorum copiam pertractari et describi non posse, nisi ab eo, qui laetiore otio et ampliore apparatu gauderet, quam quibus ego nune utor. At ne possessoris voluntati omnino deessem, illud certe mihi agendum esse putavi, ut insecta ab interitus periculo tuerer, omnia in ordine quodam disponerem, paucas coleopterorum in Africa calidiore habitantium familias accuratius percenserem, nomina eorum, quae jam descripta essent, nulla uberioris synonymiae ratione habita indicarem, nova quaedam describerem, de omnibus denique ita dissererem, ut intelligi posset, nun parum utilitatis harum rerum peritos ex is capturos esse. Quod si mihi, ut praestarem, contigit, demandatam mihi provinciam non male administravisse mihi videbor.

Itaque has pagellas benevole accipe, candide lector! Noli, quae Tibi offero, assensu Tuo omnino indigna judicare; sin autem minus placeant, certe tamen scito, officiosum me potius, quam vanae gloriolae cupidum haec pauca scripsisse.

\section{Ci c in d e 1 a e.}

1. Cicindela dorsalis. Dej.

Dej. Sp. T. II. p. 426 n. 139.*)

Symb. phys. Dec, III. n. 5. T. XXI. f. 5. ${ }^{* *}$ )

Patria: Dongola (0. Brehm). Sennaar, Abu-Harrass (A. Brehm). - Habitat etiam in Aegypto et Senegambia (Dej.).

*) Dejean Species général des Coleoptères de la collection de M. le Comte Dejean Tome I - V. Paris 1825 - 1831.

**) Klug Symbolae physicae, seu Icones et Descriptiones Insectorum, quae ex itinere per Africam borealem et Asiam occidentalem Hemprich et Ehrenberg redierunt Decas I-V. Berolini 1828 - $\mathbf{1 8 4 5}$. 
2. Cicindela fimbriata. Dej.

Dej. Sp. T. V. p. 240 n. 169.

Patria: Dongola (0. Brehm). - In Senegambia (Dej.).

3. Gicindela aegy tiaca. Klug - Dej.

Dej. Sp. T. I. p. 96 n. 79. et T. V. p. 213 no. 79.

Symb. phys. D. III. n. 7. T. XXI. f. 7.

Patria: Dongola (0. Brehm). Sennaar, Abu-Harrass (A. Brehm), - Praeterea in Aegypto, in Tanger, in Senegambia (Dej.) et in Angola (Erichs. in Wiegur. Arch. Jahry. IX. p. 204).

Annot: Cicindelam melancholicam Fabr. hujus speciei varietatem in insulis et oris maris interni indigenam Africae tropicae deesse crederem, nisi comes Dejean eam a Schoenherro ex Sierra Leona allatam sibi missam esse affirmaret (cf. Dej. Sp. T. V. p. 243 n. 171). Omnes ab Oscaro et Alfredo Brehm in Dongola et Sennaar captae ad eam formam pertinent, quae a Dejean. Cic. aegyptiacae nomine primum desccripta est.

4. Cicindela rectangularis. Klug.

Symb. phys. D. III. n. 8. T. XXI. f. 8.

Patria: Dongola (0. Brehm).

5. Cicindela regalis. Dej.

Dej. Sp. T. V. p. 251 n. 179.

Patria: Sennaar, Abu-Harrass (A. Brehm). - Habitat etiam in Senegambia (Dej.).

Annot: Haec splendidissima species septentrionem versus vix ultra gradum quintum decimnm I. s. procedit.

6. Cicindela nilotica. Klug - Dej.

Dej. Sp. T. I. p. 119 n. 101.

Patria: Dongola (0. Brehm).

Annot: Alfredus Brehm mihi narravit, interdin solis radiis ardentibus volatum cicindelarum esse rapidissimum, quare eas difficillime deprehendi. Prima luce autem, ubi somno et rore nocturno graves plantarum folis et caulibus inhaererent, se haustore (quod instrumentum entomologi Schoepfer, Koescher nominant) plantas riparias perstringentem uno tractu magnam earum copiam corripuisse.

\section{Ca $\mathbf{r} \mathbf{a} \mathbf{b}$ i.}

7. Zuphi am ruficeps. n. sp.

Patria: Sennaar (A. Brehm).

Rufum; elytris striatis, fuscis, tribus maculis rufis. Long. $4^{\prime \prime \prime}$

A Z. olenti capite rufo, antennarum articulo primo toto rufo, elytrorum sfriis paulo profundioribus pedibusque pallidioribus diversum.

8. Cymindis suturalis. Dej.

Dej. Sp. T. I. p. 206 n. 5.

Patria: Sennaar (A. Brehm). - Aegyptus (Dej. - 0. Brehm). 
9. Brachinus posticus. Dej.

Dej. ș. T. V. p. $\$ 2+$ n. 62.

Patria: Sennar (A. Brehm). - Senegambia superior (Dej.)

10. Brachinus nobilis. Dej.

Dej. Sp. T. V. p. 415 n. 52.

Symb. phys. I). III. n. 3. T. XXII. f. 6.

Patria: Sennaar (A. Brehm). - Nubia. Dongola (Dej.).

11. Graphipterus lineatus. Klug.

Symb. phys. D. III. n. 3. T. XXII. f. 9.

Patria: In Sennaar et in ripis fluminis coerulei (B. Brehm). - Habitat prope Suckot Nubiae (Klug).

12. Graphipterus Sennariensis. Laporte.

Patria: Dongola (A. Brehm).

13. Anthia maruinata. Klug - Dej.

Dej. Sp. T. I. p. 347 n. 8.

Patria: Sennaar et ripae fluminis coerulei (A. Brehm). - Nubia (Dej.)

14. Siagona A Ifredi. n. sp.

Patria: Sennaar.

Atra, nitidissima, laevis, elytris serie scrobicularum impressis. Long. $11^{\prime \prime \prime}$. Lat. 4"t $^{\prime \prime \prime}$

Species insignis, pro genere grandis, superficie paulo convexiore laevissima a reliquis hujus generis diversa.

Mandibulae breves, fortes, intus dente armatae, extus valde incurvae, apice acutae. Caput magnum, fere quadratum, laeve, carina utrinque ab autennarum insertione ad oculos porrecta instructum. Thorax longitudine duplo latior, lateribus anguste marginatus, rotundatus, pone medium valde augustatus, cordiformis, antice late emarginatus, angulis anticis acutis paulum deflexis, basi truncatus, angulis rectis, laevissimus, in medio linea satis profunda basin non attingente impressus. - Elytra thorace triplo longiora, basi angulos thoracis yix superantia, nec adeo pone medium dilatata, quin fere parallela appareant; apice conjunctim rotundata; plana, faevissima; in utroque sex serobiculae, serie propius a sutura quam a margine, aequis fere intervallis dispositae; praeterea intra humerum singula conspicitur; altera series punctorum inseulptorum a basi ad apicem proxime marginem decurrit. Antennae forma eadem, qua in caeteris; articulis quatuor primis glabris, sequentibus pubescentibus, at minus, quam in S. fuscipede aliisque. Pedum statura a caeteris vix differt, nisi articulis larsorum praesertim in primo pari longioribus et capillis fulvis in tibiarum anteriorum incisura densioribus.

15. Siagona rufipes. Fabr.

Dej. Sp. T. 1. p. 358 n. 1. et T. V. p. 475 n. 1.

Cucujus rufipes Fabricius Fnt. syst. II. 94. n. 3.

matroty Patria: Sennaar (A. Brehm). Barbaria et Europa, ubi in extremo Hispaniae meridie vivit.

16. Siagon a fuscipes. Bonelli.

Bonelli. Observations entomologiques 2.p. 26. n. 2.

Dej. Sp. T. I. p. 359 n. 2. et T. V. p. 476 n. 2.

Patria: Sennaar (A. Brehm). - Aegyptus. Senegambia. 
17. Siagona senegalensis. Dej.

Dej. Sp. T. V. p. 476 n. 10.

Patria : Sennaar (A. Brehm), - Senegambia Dej.

18. Siagona europaea. Dej.

Dej. Sp. T. II. p. 468 n. 9.

Patria: Sennaar (A. Brehm). - Aegyptus (0. Brehm).

Annot: Omnes hujus speciei Siagonae, quas 0. et A. Brehm in Sennaar et A egypto collegerunt, pubescentia densiore et longiore ab europaeis differunt. Formae autem et puncturae diversitates vel acrior inquisitio haud facile inveniet. Itaque eas ad S. europaeam adjungere non dubitavi.

19. Scarites procerus. Klug - Dejean.

Dej. Sp. T. I. p. 372 n. 5.

Symb. phys. D. III. n. 1. T. XXIII. f. 3.

Patria: Abu-Harrass (A. Brehm). - Nubia (Dej.).

20. Scarites asphaltinus. Klug.

Symb. phys. D. III. n. 3. T. XXIV. f. 1.

Patria: Abu-Harrass (A, Brehm), - Arabia deserta (Klug).

21. Clivina grandis. Dej.

Dej. Sp. T. II. p. 478 'n. 22.

Patria: Sennaar (A. Brehm). - Senegambia (Dej.)

22. Clivina rugiceps. Klug.

Symb. phys, D. III. T. XXIII. f. 6.

Patria: Dongola (0. Brehm). - Aegyptus superior et Nubia (Klug).

23. A potomus flavescens. n. sp.

Patria: Dongola. Sennaar (A. Brehm).

Flavescens, dense pubescens, pedibus pallidioribus. Long. $2^{\prime \prime \prime}$.

Simillimus quidem Apotomo rufo Oliv. elytris tamen paulum latioribus, brevius at densius pubescentibus, corporis colore praesertim pedum dilucidiore diversus esse videtur.

24. Calosoma rugosum. Degeer.

Carabus rugosus Degeer. VII. p. 627 n. 23. T. 47. f. 2.

Dej. Sp. T. II. p. 202 n. 9.

Patria: Abu-Harrass (A. Brehm). Prom. Bon. Sp. (Dej.)

25. Calosoma chlorostictum. Klug.

Symb, phys. D. III, n. 1. T, XXIII. f. 10.

Calosoma rugosum var. Dej. Sp. V. p. 558 n. 9.

Patria: Dongola (0. Brehm).

Annot: Comes Dejean hane speciem Cal, rugosi varietatem esse judicavit; at praeter habitum diversum - nam est paulo longior et angustior - accuratius perscrutanti aliae etiam differentiae se offerunt, quas celeberr: Klug, quo solet acumine, ita exposuit: , a Cal. rugoso, cuiquam maxime affinis, differre tamen videtur colore in omnibus nigro potius, quam âeneo, punctis impressis semper viridi-coeruleis, nec unquam cupreo-aeneis thoraceque confertim qui- 
dem punctatis, haud tamen scabris, elytrorum striis denique laevibus potius, quam crenatis."

Accedit, quod in sola Dongolae provincia, nec usquam alibi inventum est. 26. Colosoma imbricatum. Klug.

Symb. phys. D. III. n. 2. T. XXIII. f. 11.

Patria: Sennaar, Abu-Harrass, ripae fluminis coerulei (A. Brehm). - Praeterea Dongola (Klug).

27. Omophron tessellatum. Dej.

Dej. Sp. T. II. p. 260 n. 3.

Patria: Dongola (0. Brehm). - Aegyptus (Dej.).

28. Chlaenius transversalis. Dej.

Dej. Sp. T. V. p. 617 n. 71.

Patria: Dongola (0. Brehm). Sennaar, Abu-Harrass, ripae fluminis coerulei (A. Brehm).

Annot: 1) Comes illustris Chl. interruptum Klug, Aegypti incolam, ab ipso celeberrimo auctore nomine indicato acceptum hujus speciei varietatem esse arbitratus est.

Annot: 2) Maculae quatuor fasciam formantes plerumque cohaerent, interdum elytrorum colore disjunctae sunt; raro macula septimi intervalli omnino deest.

29. Chlaenius sulcipennis. Dej.

Dej. Sp. T. II. p. 307 n. 9.

Patria : Dongola (0. Brehin). - Nubia (Dej.).

30. Chlaenius togatus. Klug.

Symb. phys. D. III. n. 5. T. XXIV. f. 6.

Patria: Dongola (0. Brehm). Sennaar (A. Brehm).

31. Epomis circumscriptus. Dufts.

Carabus circumscriptus Duftschmidt Fanna Austriaca T. II. p. 166 n. 219.

Epomis circumscriptus Dej. Sp. T. II. p. 369 n. 1.

Patria: Dongola (0. Brehm). - Nubia, Senegambia (Dej.). Angola (Erichson. Vide ejus dissertationem de Fauna insectorum Angolensi in Wiegm. Arch. für Naturgesch. Jahro. IX. p. 205). - Italia, Gallia meridionalis (Dej.). Austria (Dufts.).

Annot: Hoc insectum in illis numeratur, quae per amplissimos terrarum tractus sparsa sunt. Nam ut a septentrione meridiem versus a Gallia meridionali per totam fere Africam usque ad regnum Angolense procedit, ita e Senegambia per interioris Africae plagam radices montium Abyssiniae attingit. In Africa calidiore paulo minoris staturae est, quam in Europa, quod jam Dejean animadvertit in Sp. T. II. p. 370.

32. Feronia conformis. Dej.

Poecilus parallelus Klug.

Dej. Sp. T. III. p. 230 n. 22.

Patria: Chartum (A. Brehm).

33. Argutor aeneus. Dej.

Dej. Sp. T. HI. p. 247 n. 38.

Var. Argutor bicolor Klug (i. I.) abdomine subtus et pedibus ferrugineis. 
Patria: Dongolir (O. Brehm). - Aegrphus (0. Brehm. Dej.)

Annot: In immaturis color pallidus et minus metalicus. Ad hos pertinet A. bicolor Kilug (i. l.) addomine pedihasque ferrugineis. Rarius etiam elytra obscure ferruginea sunt.

34. Platymetopus tessellatus. Dej.

Dej. Sp. T. IV. p. 78 n. 8.

Patria: Dongola (0. Brehm). Sennar (A. Brehm). - Senegambia (Dej.)

Annot: Macularum in elytris situs semper idem, anbitus vero et numerus rariabilis; modo una et altera deest, modo longius productie sunt ita, it strias efficiant.

35. Acupalpus disciger.n. sp.

Oblongus, rufo-testaceus; elytris striatis, pone medium macule comnuni moludu nigro-violaceu; pedibus pallidioribus. Long. $2^{\prime \prime \prime}$.

Patria: Sennaar (A. Brehm).

Unum hajus lepidissimi insecti specimen misere mutilatum ad accuratiorem descriptionem vix idoneum videatur.

36. Bembidium nil oticum. Dej.

Dej. Sp. T. V. p. 73 n. 36.

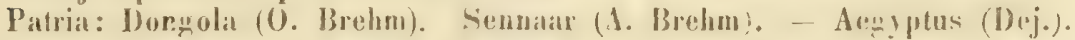

Annoi: Paulo ante maculam apicalem alia rotunda ejusdem coloris propm marginem sita conspicitur, quae, quamquam interdum olsoletion, in iis tamen, quae inspicere mihi licuit, nunquam omnino deest. Caeterum rum Depeanii descriptione congruunt. (Q) ui rognitum habent, quam facile istac matculat in $B$. pygmaco Fubr., B. gullula Gigll. et aliis hojus generis insectis obsolescant. non mimabutur, quod earum in Dejoanii descriptione nulla mentio firta est.

37. Bembidium aeg y tiacum. Dej.

B. pallipes Klng (i. 1.).

Dej. Sp. T. V. p. 90 n. 50.

Patria: Dongula (O. Brehm). - Aegyptus (0. Brehm. Bej.)

38. Bembidium Copticum.n. sp.

Nigro-piceum, paulum aeneo-micans, llowace transverso, convexo, dyfris

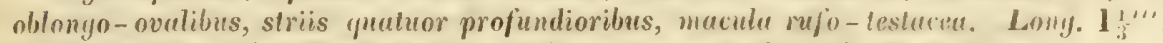

Patria: Dongola (0. Brehu). - Aeryptus superior (0. Brehum).

Nigro-piceum, nitilum rel aeneo-micans. Caput triangudare, antice lineis duabus brevibns parallelis impressum. Antennae fuseae, articulis primo et secundo rufo-testacejs. Thorax transversus, convexus, lateribus potundatus, aute marginem posticum linea impressa, utringue in foveolam excunte; elytra oblongo-ovalia, humeris obtpisis, apice rotundata, vix sinuate, striis tribus dorsalibus profundioribus, prima satuali integra, secunda ante basin et apicem albbreviata, tertia brevior alloue, quam secunda; practerea prope marginem stria quarta integra. Corpus subtus nigrum nitidum. Pedes rufo-testacei femorum basi picea.

Similis B. assimili Gyll., als quo thoracis forma ot stris paucioribus non punctatis facile discernitur. 
39. Bembidium ornatum. Klug (ined.) n. sp.

Viridi-aeneum; thorace transverso, subquadrato, utrinque bifovcolato; elytris punctato-striatis, maculis in utroque duabus, altera pone humerum oblonga, altera ad apicem lunata, flavis. Long. 2."

Patria: Dongola (0. Brehm). - Aegyptus superior (0. Brehm).

B. statura Amarae parvae (B. amaroides Kollar?), viridi-aeneum; caput triangulare laeve, duabus striolis juxta oculos brevibus parallelis; ore et palpis flavo-testaceis; antennarum articulis quatuor primis flavo-testaceis, sequentibus obscurioribus, pubescentibus. Thorax subquadratus, marginilous ante medium paulo dilatatus, margine antico emarginatus, angulis anticis obtusis, basi lisinuatus, anæzulis posticis acutis, intra basin transversim impressus foveolisque duabus exsculptus; linea media longitudinalis marginem anteriorem non attingit. Elytra oblongo-ovata, ad basin thorace lateriora, punctato-striata, striis septem ornata, suturali integra, quatuor sequentibus basi apiceque abbreviatis; sexti loco punctorum series brevissima conspicitur; septima ad apicem cum marginali conjuncta est; brevis striola profunda als apice excurrens quintae striolae dorsalis rudimentum esse videtur; in utroqne macula oblonga pone humerum, altera ad apicem lunata flava. Corpus subtus piceum, nitidum. Pedes pallidi.

\section{Dytis ci.}

40. Cybister africanus. Laporte.

Lap. Etud. ent. p. 99.

Patria: Sennaar (A. Brehm). - Tota Africa, Sicilia, Sardinia, Hispania (Aubé).

41. Eunectus sticticus. Linné.

Dytiscus sticticus Linné. Systema Naturae 11. p. 666. n. 12. Fabr. E. S. 'T. I. p. 191 n. 16.

Dytiscus griseus Fabr. E. S. T. I. p. 191 n. 17.

Annot: Fabricius primus formam genuinam in E. S. D. grisei nomine edidit. Vereor autem, si clar. Aubéi rationem sequens hoc nomen praevalere julıeam, ne a legibus de nominum auctoritate praescriptis recedan.

Omnes fere hujus speciei dystisci in Dongola, Sennaar aliisque regionibus australioribus capti ad formam pertinent, quam clar. Klug in Symbolis physicis E. succincti nomine publicavit. Pauci tamen thoracem fsacia nigra in medio vel angustata, vel interrupta, ornatum habent.

In magna horum insectorum eopia ab $A$. et 0 . Brehm collecta ne unum quidem exemplum E. h elvoli Klug (Symb. phys. D. IV. n. 2. T. XXXIII. f. 3.) integrum conservatum est. Exstant tamen duo trunci capite cum thorace mutilati, quos in hac specie numerandos puto.

42. Hydaticus signatipennis. Laporte.

Lap. études entom. p. 95.

Aubé Hydrocanth. p. 158 n. 2*).

*) Aubé, Species général des Hydrocanthares et Gyriniens. Paris 1838. 
Patria: Ripae fluminis coerulei (A. Brehm). - Senegambia (Aubé). ordofan, Angola, Madagascar, India orientalis (Erichson F. Angol. in Wienm. Arch. Jahrg. IX. p. 205),

43. Hydaticus Leander. Rossi.

Rossi Fauna Etrusea I. p. 212.

Patria: Abu-Harrass (A. Brehm), - Aegyptus (0. Brehm). Senegambia, Europa australis (Anbé).

44. Hydaticus bivittatus. Laporte.

Lap. études entom. p. 97.

Patria: Abu-Harrass (A. Brehnı). - Senegambiri, Madagascar, Isle de France (Aubé).

45. Hydrocanthus guttula. Aubé.

Aubé Hydroc. p. 410 n. 6.

Patria: Sennaar (A. Brehm). - Madlagasear, Isle de France (Aubri).

46. Hy drop orus Solieri. Aubé.

Aubé Hydroc. p. 554 n. 57.

Patria: Sennaar (A. Brehm). - Aegyptus (Aubé).

47. Hydroporus turgidus. Erichson.

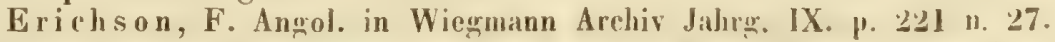

Patria: Sennaar (A. Brehm). - Aegyptus (0. Brehm Mius. Ber.). Ingola (Erichson 1. c.).

48. Hydroporus signatellus. Kilug.

Symb. phys. D. IV. n. 5. T. XXXIV. f. 3.

Patria: Sennaar (A. Brehm), - Dongola (Kilug).

\section{G y $\mathbf{r}$ in i.}

49. Gyrinus distinctus. Aubé.

Aubé Icon. p. 385 pl. 43. f. 3.

Patria: Abu-Harrass (A. Brehm). Dongola (O. Brehm). - Aegyptu(0. Brehm. Aubé). Nec frigidiores Europae aquas abhorret. Eitenim si Gyr. colymbus Erichson eadem species est, ynod hodie inter omnes entomologos constat, septentrionem versus aden ad Berolini lacus adscendit. Cf. Erichson: Die Küfer der Mark T. I. p. 191 n. 2.

50. Orectocheilus sericeus. Klug.

Symb. phys. D. IV. n. 6. T. XXXIV. f. 12.

0. palliatus Klug. - Dejean Catal, ed. 3. p. 67.

Patria: Ripae fluminis coerulei (A. Brehm). Dongola (0). Brehum). Aegyptus (0. Brehm). Nubia (Aubé).

51. Orectocheilus glaucus. Klug.

Symb. phys. D. IV. n. 5 T. XXXIV. f. 11.

Patria: Sennaar (A. Brehm). Dongola (0. Brehm). - Aegyptus (0. Brehun). 
52. Orectocheilus Oscari.n. sp.

Oblongo-ovatus, oonvexus, brunneus, sericeo-pubescens, huteo-marginutus; clytris costa sulurali laevissima ante apicem abbreviatu; angulis apicis externis in spinam productis, internis acutis. Long. 4".".

Patria: Dongola (0. Brehm).

0 . sericeo KI. sinilis, at paulo minor et angustior; thoracis laterihus, elytris subtilissime punctatis, striis eorum puhescentibus vix couspicuis; costa a) Jytrorum suturali, cujus margo exterior sinuatus, postice angustiore ante apicem abbreviata praesertim al, illo distinctus.

53. Vineutes subspinosus. Klug.

Gyrimus subspinosus Klug symb. phys. D. IV. n. 3. T. XXXIV. f. 9.

Patria: Alu-Harrass (A. Brehm). - Aegyptus (0. Brehw). Nubia, Senegambia, Madagascar, Isle de France, India orientalis (Aubé). Mauritius, Angola (Erichson F. Ang. in Wiegm. Arch. Jahrg. IX. p. 206).

\section{Mi i s t i.}

34. Hister gigas. Paykull.

Payk. Monogr. p. 9 n. I. $\left.^{*}\right)$

Patria: Abu-Harrass (A. Brehm). - Senegambia (Payk.).

55. Hister nigrita. Erichson.

Erichs. Histeroid. in Klug Jahrb. p. 131 n. 7.

Patria: Sennaar (A. Brehm). - Senegambia (Erichs.)

56. Hister tropieus. Payk.

Payk. Monogr. p. 18 n. IX.

Patrin: Dongola (0. Brehm). - Guinea (Park.). Nubia (Erichs.).

57. Hister impressus.n. sp.

Aler, nitidus, ovalus, thorace utringue bistriato, striis interiore paulo ant: basin, exteriore pone medium abbreviatis, elytris striis dorsulibus duabus primis ubbreviatis, segmentis abdominis duabus ultimis punctis profundioribus mimus confertis, penullimo utrinque impresso. Long. $\mathbf{4}^{\prime \prime \prime}$.

Patria: Sennaar, Ahu-Harrass (A. Brehm).

Ater nitidus. Frons parte anteriore alorupte angustata, stria ordinaria satis profunda; labrum a fronte impressione distinctum, parvum, transverse ellipticum; mandibulae capitis longitudine, dentatae. Thorax longitudine triplo latios, laevis, convexus, margine antico emarginato, lateribus vix rotundatus, margine: postico truncato, angulis anticis acutis dellexis, posticis rectis, stria interiore integra cum stria secundum marginem anticum producta ita conjuncta, ut linea continua per totum thoracis ambitum decurrat; stria exteriore pone medium ahbreviata, ad angulum anticum intus reflexa in lineam marginalem reclinata. Elytra thorace dimidio longiom, basi thoracis latitudine, pone humeros paulum dilatata, rersus apicem angustata, striis dorsalibus primo in medio, secunda

*) Paykull, Monographia Histeroidum. Upsaliae 1811. 
pone medium abbreviatis, reliquis quatuor integris; stria marginali exteriore nulla, interioris locum occupat brevissima tantum linea in medio inter sextam striam et marginem sita; fossa lateralis carina in duas partes divisa, quarum latior rugis transversis in areas quadratas divisa est.

Penultimum aldominis segmentum utrinque impressum, punctis majoribus praesertim versus marginem profunde impressis; segmentnm ultimum simili modo insculptum, punctatum, puncta tamen praesertim in summo apice minora et minus profunda.

Tibiace pedum anterioriam dilatatae, tridentatate, posteriores in margine exteriore duplici spinarum ferruginearum serie armatae. Corpus subtus picenm, nitidum,

58. Hister memnonius. Erichson.

Erichson Histeroid. in Klug Jahrb. p. 134 n. 11.

"Suborbiculuris, ater, nilidus, mandibulis dentatis, thorace utringue subunistriuto, alylris stria dorsali prima obsoleta, secunda abbrevinta, tibiis anticis tridentatis. Long. $4 \frac{1}{2} "$ " E Erichson.

Patria: Dongrola (0. Brehm).

59. Hister bimaculatus. Linné.

Linné Systema Naturae 11. p. 567 n. 5.

Patria: Dongola (0. Brehm). … Asegyptus superior (0. Brehm). Indiat orientalis (Payk.). Tota Europa.

(i0). Saprinus chaleites. llliger.

Illig. Mag. T. VI. p. 40 n. 15.

Patria: Dongola (0. Brehu). - Aegrvptus superios (0. Brehm). Enropat australis. 

Sieben und vierzigste Nachricht von de m

\title{
Friedrichs - Gymnasium
} zu Atenburg auf das Schuljahr Ostern 1853 bis dahin 1854.

\author{
A Is \\ Einladungsschrift \\ zu den \\ rom 3. bis 7. April 1854 \\ in der Aula des Josephinum \\ stattfindenden

\section{Schulfeierdichlocitem von} \\ Schulrath D. formtid) EDuard fows, \\ Director.
}

Beigrefiigt ist eine Abhandlung von Prof. J. H. A petz: De Coleopteris, quae Oscarus et Alfredus Brehm in -Afriea legerunt.

\section{A I. T IN B U $\mathbf{R}$,}

gedruckt in der Hofbuchdrackerei. 


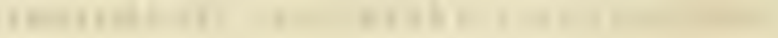

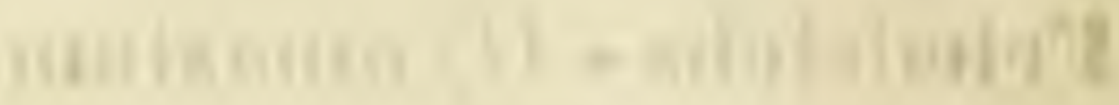

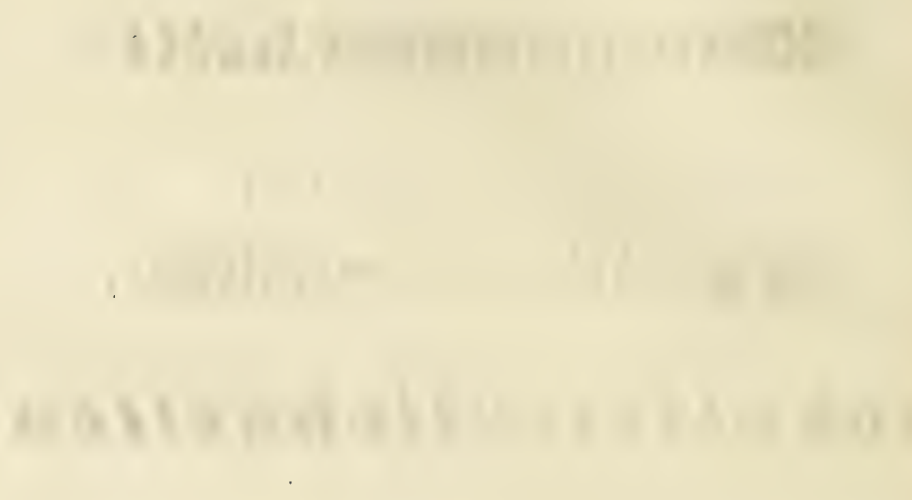

, 


\section{Ordnung der Schulfeierlichkeiten.}

Die öffentliche Prüfung der Schüler des Gymnasiums wird vom 3. bis 6. April in nachstehender Ordnung abgehalten werden.

Montag, den 3. April Vorm. examiniren in Mittel-Secunda:

8-9 in der Naturgeschichte Hr. Prof, A petz.

9 - 10 im Lateinischen Hr. Prof. Lorentz.

10 - 11 im Griechischen der Director.

Nachm. in Unter-Secunda:

$2-2 \frac{3}{4}$ im Lateinischen Hr. Prof. Zetzs che.

$23-32$ in der Mathematik Hr. Prof. Braun.

$3 \frac{1}{2}-4 \frac{1}{4}$ im Griechischen Hr. Prof. Lorentz.

Dinstag den 4. Vorm. in Ober-Secunda:

8-9 in der. Religionslehre Hr. Prof. Frank.

9 - 10 im Lateinischen Hr. Prof. A petz.

$10-11$ im Griechischen Hr. Prof. Zetzsche.

Mittwoeh den 5. Vorm. in Prima:

8-9 im Griechischen Hr. Prof. Zetzsehe.

9- 10 in der Physik Hr. Prof, Braun.

10-11 in Lateinischen Hr. Prof. Frank.

Nachm. in Selecta und Prima:

$2-3 \frac{1}{2}$ (Selecta $2-2 \frac{3}{4}$, Prima $2 \frac{3}{4}-3 \frac{1}{2}$ ) im Französischen Hr. Prof. Köhler.

$3 \frac{1}{2}-4 \frac{1}{2}$ im Hebräischen Hr. Prof. A petz.

Donnerstag den 6. Vorni. in Selecta:

8-9 in der Religionslehre Hr. Prof. Frank.

9 - 10 in der Mathematik Hr. Prof. Braun.

10 - 11 im Lateinischen der Director. 
Freitag den 7. April Vormittags von $10 \mathrm{Uhr}$ an: Translocation.

Nach einem kurzen Gesange und einer einleitenden Rede des Herrn General-Superintendenten Dr. B ra u ne als Ephorus des Gymnasiums findet die Bekanntmachung der Translocation und die Vertheilung der Prämien durch den Director Statt.

Hierauf: A biturienten-Entiassung.

Nach der Bekanntmachung der deu einzelnen Abiturienten ertheilten Consistorialdiplome durch den Herrn Ephorus wird Rudolph Wilhelm Schulze aus Altenburg im Namen aller zur Universität Abgrehenden in einer lateinischen Rede von der Anstalt Abschied nehmen, und der Selectaner F riedrich Hugo Richard Wimmer aus Altenburg im Namen der Zurückbleibenden darauf antworten. Hierauf Entlassungsiede des Directors.

Zu diesen Schulfeierlichkeiten lade ich die verehrungswürdigen Mitglieder des Geheimen Ministeriums und der Landescollegien, so wie alle Gönner und Freunde des Schulwesens überhaupt und unserer Schule inbesondere hiermit ehrfurchtsvoll und ergebenst ein.

\section{Schulchronik.}

Das Schuljahr 18 $\frac{52}{5}$ wurde an 18. März v. J. in gewohnter Weise mit der Entlassung der Abiturienten geschlossen. Bei dieser Feierlichkeit erhielt der Abiturient $\mathbf{0}$ skar $\mathrm{H}$ ug o Braun aus Altenburg die Lingke'sche Prämie. Die Abiturienten Richard Geutebrïck und Max Alexander Dietz, heide aus Altenburg, welche neben ihm dem hochverehrten Stifter und gegenwärtigen Collator der Prämie, Hrn. Finanzrath Lingke, durch das Lehrercollegium präsentirt worden waren, empfingen von demselben zum Andenken an den für sie so wichtigen Tag ihrer Entlassung von der Schule jeder einen goldenen Siegelring.

Die v. Lindenau'schen Prämien wurden den Abiturienten 0 skar Hugo Braun und Max Alexander Dietz ertheilt.

Die Bücherpränien, welche die hiesige Loge mil gewohnter Liberalität dem Lehrercollegium zur Vertheilung zugewiesen hatte, emptingen die Selectaner Steinbach, Saupe, Leipold, die Primaner Eckardt, V. Reichard t, A. Wagner, der Oher-Secundaner Quas, der Mittel-Secundaner C. Steiniger, die Unter-Secundaner Günther und B. Kellberg.

Die v. Breitenbauch'schen Stipendien erhielten die Selectaner Bräutigam, Scherff, Knipfer und Schiffmann, die Primaner Böttger und Stïnzner.

Das Schuljahr 18 $\frac{5}{5} \frac{3}{4}$ wurde Montag den 4. April nit der Prüfung der neu angemeldeten 20 Schüler begonnen. Am folgenden Tage nahmen die Lectionen in allen Classen ihren Anfang. Die Erbauungsrede, welche dem Beginn des Unterrichts voranging, hielt $\mathrm{Hr}$. Prof. Huth. 
Am 16. Mai verloren wir durch den Tod einen lieben Sthuiler, den Selectaner Bernhard Hempel aus Altenburar. Er war ein trefllicher, durch Fleiss und Sittenreinheit ausgezeichneter Jüngling, dessen glïckliche Geistesgahen sich zur Freude seiner Eltern und Lehrer immer sichibarer entwickelfen, und dessen Aufenthalt nuf dem Gymnasium ein stetes Vorwärtsstreben narh dem Bessern genannt werden konnte. Sein Tod, der nach längerer Krankheit erfolgte, erwerkte deshalb die allgemeinste Theilnahme und wurde besonders auch von seinen bisherigen IJehrern und Mitschülern tief empfunden. Doch den Trost wenigstens hatten diejenigen, die ihn im Leben liebten, dass er von der treuesten I,iebe theurer Eliern behütet, von Jer zärtlichsten Sorwfalt geliebter Verwandten umgelsen, ruhig und schmerzlos geendet hatte. Was ein Mutterherz an Liebe gewähren kann, das ist ihm zu 'Theil geworden und hat seine letzten Tage verschönt, so dass er ohne Ahnung des nahenden Todes, mit heitern Plänen für die Zukunft beschäftigt von dannen schied. Er ruhe in Frieden!

Aun 22. Mai wurde dem Lehrercollegium das hohe Glück zu Theil, Sr. Hoheit dem Erluprinzen Ernst und Threr Hoheit der Erloprinzessin Ag nes als Hohen Neuvermählien nach Höchstihrem Einzuge in Alienburg durch eine Deputation, bestehend aus den Professoren Apetz, Braun und in ir, seine ehrerbietigsten Glückwünsche auszusprechen und einen von Hrn. Prof. H uth gedichteten ,Festgruss" zu überreichen.

Am 24. Mai war es den Schülern des Gymnasiums gestattet, den hohen Neuvprmihlten in rinem solennen Fackelzuge ihre Huldigungr datubringen und durch eine aus den Selectanern B r ä u tiga m, S ch u I ze und K $\mathrm{ni}$ fe $\mathrm{r}$ bestehende Deputation ein beglüekw ünsehendes Gedicht zu ïberreichen.

Am 30. September hörte Hr. Prof. H uth auf, dem Gymnasium als Lehrer anzugehören, da er zam Ephoralverweser und Stiftspredigner in hiesiger Stadt ernannt worden war. Sein Abgang was ein scluwerer Verlust fïr die Schule, und Lehrer sowohl wie sichüler salien ilın mit grossem and aufrichtigem Bedauern aus ihrer Mitte scheiden. Fast 24 Jahre ist dieser allgemein hochgeachtete nud verehrte Mann mit der gewissenhaftesten Treue, mit unermüdlichem Eifer, mit sichtbar segensreichem Erfolge an dem Grmnasinm für die religiöse, sittliche und geistige Ausbildung seiner Schüler thälig gewesen, und noch glaubten wir, da er noch in den Jahren rüstiger Kraft steht, auf eine linge Wirksamkeit von ihm an unserer Anstalt rechnen zo können, als er den Entschluss fasste, seine Kråfte einem andern Berufe zu widmen. So übprraschend und schmerzlich uns aurh dieser Entsrhluss war, so mussten wir dorh die wohlerwogenen Gründe desselben ehren. Die Schüler des Gymnasiums hrachten ihrem verehrten Lehrer am 29. Septemher ein Stïndchen und sprachen ihm ihren Schmerz über sein Scheiden aus. Unsere herzlichsten Wünsche begleiteten ihn in seinen neuen Wirkungskreis; in unsern Herzen bleibt ihm das freundlichste und dankbarste Andenken bewahrt, und in den Annalen der Schule wird sein Name stets mit hohen Ehren genannt werden. 
Am 13. October wurde der bisherige Subrector Dr. Frank aus Ratzeburg, ein ehemaliger Schüler des hiesigen Gymnasiums, der an Stelle des Hrn. Prof. Huth als sechster Professor an die Anstalt berufen worden war, durch den Ephorus des Gymnasiums Hrn. Generalsuperintendent Dr. B ra un e in sein neues Amt feierlich eingeführt. Am 17. desselben Monats trat er seine Stelle an, inden er bis zum Schlusse des Schuljahres die Stunden seines Vorgängers sämmtlich ïhernahm. Von Ostern an wird er die Stellung eines Religionslehrers am Gymnasium einnehmen, da ihm durch die hohen und höchsten Behörden bei seiner Berufung (ausser dem dentschen Unterrichte in Selecta) der Religionsunterricht in allen Classen und der Unterricht im Hebräischen übertragen worden ist.

Am 20. October wurde zur Feier des Gedächtnisses Sr. Hoheit des verewigten Herzors Georg zu Sachsen-Altenburg, durch dessen am 3. August erfolgtes Ableben auch das Friedrichs-Gymnasium in die tiefste Trauer versetzt worden war, in der Aula des Josephinum ein Redeactus gehalten, bei welchem zuerst Hr. Prof. Lorentz die Gedlächtnissrede auf den Hochseligen Gönner und Beschützer unserer Anstalt hielt, worauf eine lateinische Gedächtnissrede des Selectaners $R u d o l p h$ Wilhelm Schulze aus Altenburg folgte. Dann hielt der Selectaner Justus Adolph Bräutigam aus Lucka eine deutsche Rede über das Thema: Das Andenken des Gerechten bleibt im Segen. Zum Schluss trug der Selectaner Julius Knipfer aus Remsa ein deutsches Gedicht: Trost in Thränen, vor.

Am 13. Februar traf das Gvmnasium ein neuer, schwerer und unerwarteter Schlag, indem unser theurer College Prof. A pel nach kurzem Krankenlager uns durch den Tod entrissen wurde. Sein Ableben erregte nicht bloss im Kreise der Schule, sondern auch in weitern Kreisen die innigste Theilnahme, das schmerzlichste Bedauern, denn er besass die allgemeinste Liebe und Achtung, und er verdiente sie. Er war ein freundliches, trenes Gemüth, welches sich leicht anschloss, aber nicht leicht das wieder anfgab, was es einunal in Liebe umfasst hatte, sondern unverändert daran festhielt. Musterhaft war er in seinem Verhältnisse zur Schule. Gewissenhafteste Pünctlichkeit, unverdrossener Eifer, unermïdlicher Fleiss, pädagogischer Tact und Geschicklichkeit, umfassende Kenntnisse zeichneten ihn aus; Freundlichkeit und Milde in der Behandlung der Schüler; lebendiges Interesse für alles Schöne und Edle, wissenschaftlicher $\operatorname{Sin} n$ wrikten veredlend und anregend auf das jugendliche Gemuith und gewannen ihm Vertramen und Liebe. Mit vollem Rechte wurde in einem von den Schülern des Gymnasinms ihm gewidmeten Nachrufe von ihm gesagt: Einen treueren Lohrer gab es nie. Wir, seine Collegen, verloren an ihm einen Freund, dessen Gedächtniss wir stets in Ehren halten werden. Durch sein Dahinscheiden ist das schöne Verhältniss, das uns mit ihı verband, zerrissen, und eine Lücke in unserm Kreise entstanden, die nicht leicht wieder auszufüllen sein wird. Am 16. Februar wurde er feierlich beerdigt, und Lehrer und Schüler sandten 
ihm, als letzten Liebesbeweis, eine Handrall Erde in die Gruft nach, die seine irdische Hülle aufnahnı. Friede seiner Asche!

Am 20. September v. J. und am 21. März d. J. feierten die Lehrer und Schüler des Gymnasiums gemeinschaftlich das heilige Abendmahl.

\section{Lehrverfassung.}

In der Lehrverfassung des Gymnasiums ist keine Veründerung vorgegangen; wegen des Todes des Hrn. Prof. Apel jedoch sind mehrere Pensa, namentlich die Geschichtspensa der beiden obern Classen, nicht ganz vollendet worden.

\section{Fünfte Classe (Unter-Secunda).}

Latein, 9 St. Davon 5 St. Grammatik. Die granze Formenlehre von Anfang an bis zu den unregelmässigen Verbis (einschliesslich). In der Syntax: die allgemeinen Regeln über die Bildung einfacher Sätze, die Erweiterung derselben durch Casus, Präpositionen, Adrerbia und über den Gebrauch der Casus bei Mass-, Zeit - und Orisbestimmungen, nelsst Erklärung der Construction des Acc. c. inf., ut, quod, der ablut. abs. Alle 8 Tage wurden Exercitien geschrieben, auf deren Correctur 1 St. verwendet wurde.

4 St. Jacobs lat. Lesehuch I. Cursus, Abschn. I. und Abschn. II. S. 3-36. Cornel. Nep. Cimon und Lysander. Hr. Prof. Z etzsche.

Griechisch, 4 St. Formenlehre bis zu den Verbis auf $\mu$ (einschliesslich), namentlich auch Einübung der Regeln über die Fintheilung und Veränderung der Buchstaben, sowie über die Formation und den Accent bei den Declinationen und Conjugationen. Auswendizlernen von Vocabeln. Einübung der Formenlehre nach Jacolss griech Lesebuch I. Cursus. Uebersetzen aus Jacolos II. Cursus, Anekdoten V und VI. Hr. Prof, Lorentz.

Deutsch, 4 St. Uebungen im Lesen, Erzählen und Recitiren von Gedichten und prosaischen Musterstücken aus dem deutschen Lesebuche. Einühung der Orthographie nach Heyse's Schulgrammatik, Formenlehre bis zu den Zeitwörtern (einschliesslich); Bildungr einfacher Sätze. Alle 14 Tage schriftliche Aufsätze. Hr. Prof. Apel.

Französisch, 1 St. Formenlehre bis zu den Hülfszeitwörtern (einschliesslich) and Einübung derselben darch schriftliche und mündliche Aufgaben. Lesen einschlagender Abschnitte aus Müller's Lespluch. Hr. Prof. Köhler.

Religionslehre, 3 St. In 2 St. wurde die rhristliche Sittenlehre vorgetragen und die zugehörigen Bibelstellen auswendiy gelernt. 
1 St. Anleitung zur Kenntniss der bihlischen Schriften überhaupt und der des A. T. insbesondere. Dann Lesen und Erklärung ausgewïhlter Abschnitte aus den historischen Schriften des A. T. Hr. Prof. Apel.

Arithmetik, 3 St. Znerst kurze Einleitung in die Arithmetik (Grösse, Eintheilung der Grössen, Einheit, Menge, Zahl, Eintheilung der Zahlen). Sodann: die rerschiedenen Zahlen - und Ziffersysteme und Aufuaben darïber. Die vier Species. Die gemeinen Brüche. Eintheilung, Verwandlung, Heben dersellsen; Primzahlen und Primzahlen unter sich; Regeln über das Erkennen des gemeinschaftlichen Divisors. Die vier Species der gremeinen Brüche. Doppelbrüche. Kettenbrïche. Decimalbrüche. Verwandlung der gemeinen Brïche in Decimalbrüche. Sämmoliche Lehren wurden durch Auflösung von Aufgaben und ein mit dem ['nterrichte fortwährend verbundenes Tiffelrechnen eingeübt. Hr. Prof. B rau n.

Geographie, 2 St. Zuerst als Einleitung das Wichtigste aus der mathematischen und physischen Geographie; sodann politische Geographic von Asien und Afrika. Hr. Prof. Zetzsehe.

Naturbeschreibung, 2 St. Botanik und Zoologie, mit Ausschluss der Gliederthiere. Hr. Prof, A petz.

Zeichnen, I St. Hr. Prof. Dietrich.

Schreiben, 2 St. Hr. Cantor $\mathrm{Ne}$ efe.

\section{Vierte Classe. (Mittel-Secunda).}

Latein, 8 St. Davon 4 St. Grammatik. Repetition des Pensum ron Unter-Secundil [1 St.]; die allyemeinen Regeln ülser das Adjectivum, die Numeralia, Pronomina, dis Verhum, Adverbium, über Erklärungs - und Causalsätze [2 St. $]$; Correctur wöchentlicher Exercitia (1 St)

4 St. Caesar de bello Gallico lib. I. mit schriftlicher Uebersetzung. Zuletzt die allgremeinen Regeln über die Quamität. Zwei- und dreisylbige Versfïsse. Sthema des Hexameters und Pentameters. Cäsur. Einübung der Regeln und des Scandirens. Hr. Prof. Lorentz.

Griechisch, 5 St. Davon 3 St. Grammatik. Die Formenlehre wurde von Anfang an nochunals durchgegangen und eingeübt, so dass die besonderen Regeln und wichtigen Ausnahmen mitgenommen wurden; sodann die unregrelmaigsigen Verba, nach ihrer Bildung in Classen eingetheilt.

2 St. Aus Lucian's ausgewählten Gespriiehen von A, Matthiä wurde S. $1-18$ und S. $80-95$ gelesen. Directar. 
Deutsch, 3 St. Lehre von der Bildung einfacher und erweiterter Sïtze, von untergeordneten und nclsengeordneten Sätzen und ron der Interpunction. Alle 14 Tage wurde eine Ausarbeitung geliefert, welche nach der Correctur öffentlieh in der Stunde durchgegangen wurde. Lese - und Declamationsïbungen nach Apel's deutschem Lesebuche. Hr. Prof. Lo rentz.

Französisch, 2 St. Formenlehre, und zwar namentlich die regelmässigen, unregelmissigen und defectiven Verlat, die Adverbion und Praepositionen. Einübungr derselhen durch Uehersetzung von Aufgaben aus der'M üller'schen (irammatik und durch Lectïre entsprechender Abschnitte aus Müller's Leseluch. Hr. Prof. Köhler.

Religionslehre, 3 St. Davon '2 St. Erklärung der Hauptlehren der christlichen Siltenlehre nebst Auswendig̨lernen von zugehörigen Bibclstellen.

1 St. Bibelkunde. Lesen und Erklärung ausgewällter historischer Stellen des A. T. Hr. Prof. A pel.

Mathematik, 3 St. A rithmetik: Practische Arithmetik, Proportionslehre und Regula de tri: Reductions-, Ketten - und Pepartitionsrechnung. Geometrie: Einleitung in die Geometrie. Erklärung aller in den Elementen dieser Wissenschaft vorkommenden Begrilhe. Erklärung der verschiedenen Lage der Linien und Ebenen im Raume und der in der Stereometrie vorkommenden Körper. Die Lehren der Arithmetik wurden durch Aufloisung von Aufgatoen und ein mit dem Unternchte verbundones 'Tafelrechnen, die der Geometrie duch Fergleichung geometrischer Formen, Ausführung den Gegenstand betreflender Constructionen und Anfertigung geometrischer Körper eingenibt und erläutert. Hr. Prof. B raun.

Geographie, 2 St. Politische Geographie von Furopa. Kartenzeichnen Hr. Prof. Zetzsehe.

Geschichte, 1 St. Die Haupthegebenheiten der Welgeschichte nach den Bredow'schen Geschichtstabellen. Hr. Prof. Lorentz.

Naturbeschreibung, 2 St. Gliederthiere und Mineralogie. Hr. l'of. A petz.

Zeichnen, 1 St. Hr. Prof. Dietrich.

Sebreiben, 2 St. Hr. Canter Neefe.

\section{britte Classe (Oher-Secunda).}

I. atein, 8 St. Davon 3 St. Grammatik. Die allgemeinen und besondern Regeln ïlser Adieclivum, Numeraliu, Pronomina, Verbum, Adverbiam. E klärungs - und Causalsätze, Die Regeln über die andern 
Arten von Sätzen. Alle 8 Tage ein Exercitium, wobei fortwährend auch auf Formenlehre und Casusreareln Pücksicht genommen wurde. 3 St. Curtius lib. V. Cic. Laelizs. Cursorisch: Caesar de bello civili lib. I. Hr. Prof. A petz.

2 St. Ovid. metumorph. lib. I, 1-415. II, 1-332. III, 1-130. $I V, 563-789 . V, 1-249.346-571$. VI, 146-312. Daneben wurden die prosodischen Regeln nochmals durchgegangen und das elegische Versmass durch Ordnen versetzter Verse eingeübt. Im Sommerhalbjahr: Hr. Prof. H u th. Im Winterhalbjahr: Hr. Prof. Frank.

Griechisch, 6 St. Davon 2 St. Grammatik. Repetition der unregelmässigen Verba. Die Regeln über die attributiven Bestimmungen, über die Congruenz, über den Artikel und die Casus. Alle 14 Tage wurde ein Exercitium anfgegeben und durchgegangen.

2 St. Xenoph. Anabasis, lib. V. c. 7. his lib. VI. c. 4. mit schriftlicher Uebersetzung. Hr. Prof. Z etzsche.

2 St. Homer. Odyss, lib. XV und lib. XVI. Vorausgeschickt wurde eine allgemeine Uebersicht über die Eigenthümlichkeiten des epischen Dialects. Hr. Prof. Lorentz.

Deuisch, 3 St. Lehre vom Periodenbau und Metrik. Alle 14 Tage wurde ein Aufsaiz geliefert. Lectüre und Erklärung prosaischer und poëtischer Musterstücke aus dem deutschen Lesebuche. Recitationsübungen. Hr. Prof. A pel.

Französisch, 2 St. Syntax. Lehre von der Wortstellung im einfachen, zusammengezogenen und zusammengesetzten Satze. Jehre von der Concretion, vom Artikel und vom Gebrauch der Casus. Einige Gallicismen und Wörterfamilien. Die zugehörigen Aufgaben der Vïller'schen Graumatik wurden schriftlich oder mündlich ïhersetzt. Gelesen wurden ansgewihlte Stücke aus Wildermuth's Chrestomathie, II. Cursus. Hr. Prof. Köhler.

Religio nslehre, 2 St. Die christliche Sittenlehre nach Niemeyer §. 1-117, verbunden mit dem Etlernen der Hauptheweisstellen. Hr. Prof. Huth. Im Winterhalbjahre: Christliche Sittenlehre, auf Grund der Bergpredigt und anderer erangelischer Abschnitte. Hr. Prof. Frank.

Mathematik, 3 St. Arithmetik: Einfache und zusammengesetzte Reductionsrechnung, die Alligations-, Zins-, Rabatt - und Discontorechnung. Die Lehre ron den entgegengesetzten Grössen und dem Gebrauche der Einschliessungszeichen in der Arithnetik.

Ge ometrie: Erklärung der verschiedenen in der Geometrie vorkommenden Sätze und Beweisarten; die gemeine Geometrie bis zur Lehre vom Kreise. Sämmtliche Lehren wurden durch entsprechende Aufgaben eingeübt. Hr. Prof. Braun.

Physik, 2 St. Nach einer Einleitung in die Physik wurden die Lehren 
ron den allgemeinsten Körperphänomenen, die Statik und Mechanik fester Körper vorgetragen und durch Zeichnungen, Beispiele und Aufgaben erläutert und eingeübt. Hr. Prof, B raun.

Geschichte, 3 St. Alte Geschichte mit Ausschluss der römischen, nebst der dazu gehörigen Geographie. Hr. Prof. Lorentz.

Zeichnen, 2. St. Hr. Prof. Dietrich.

Schreiben, 1. St. Hr. Cantor Neefe.

\section{$\mathrm{Z}$ weite Classe (Prima).}

Latein, 9 St. Daron 3 St. Grammatik. Wiedrholung der Syntax, namentlich des Cursus von Ober-Secunda. Die Lehre von der Wortstellunar, vou Periodenbau und ron der Veredlung des Ausdrucks. Alle 14 Tage ein Exercitium oder Extemporale; seit Weilnachten dafïr hleine freic Ausatbeitungen. Im summer: Hr. Prof. Huth; im Winter: Hr. Prof Frank.

4 St. Cicero mo Sulla. IIr. Prof. II uth. Cursorisch: Ausgewählte Briefe von Cicero (aus der Zeit seiner Verbannung bis zum Ausbruch des Bürgerkrieges.) Hr. Prof. Frank.

2 St. Virgil. Aen. lib. XI. Hr. Prof. II uth. Virgil Acn. Tib. XII lib. I, 1 - 140. Hr. Prof. Frank.

Griechisch, 6 St. Davon 1 St. (rammatik. Die Lehre rom Infinitiv and Participium, von den Negationen und dem Gebratuch der Modi nach Conjunctionen. Die Regeln wurden theils durch Beispicte in der Stunde, theils dureh Exercitia pingeuibt, die in der slunde durchgegangen wurden. Alle 14 'Tage ein Exercitium.

3 St. Xenoph. Cyropaed. lib. II. I) ann (eursoriseh) Arriun, expedil. Alex. lib. I c. 17 - lib. II c. 6. Hr. Prof. Zetzsche.

2 St. Homer. Ilias lib. XVII. - lib. XXIV. und lib. $\boldsymbol{V}$. Hr. Prof. A petz.

Deutsch, 3 St. Kurzer Abriss der Poetlk, verbunden mit Lectïre ron Musterstellen deutseher Schriftsteller zur Lirkläung des Vorgetragenen. Alle 4 Wochen wurde eine deutsche Ausarbeitung geliefert. Freie Vortriage. Hr. Prof. Apetz.

Französisch, 2 St. Syntax bis zur Lehre vom Infinitiv (einsclolipscliclo). Einühun der syntactischen Rexpeln nach Mäller's Grammatik und Exercitien. Gelesen wurde Michel Perrin von Melescille und Duveyrier. Hr. Prof. Köhler.

Hebräisch, 2 St. Die Formenlehre wurde durchgegangen, die Paradigmen gelernt und zur Uebung im Analysiren einige Cinpitel der Genesis gelesen. Hr. Prof. A petz. 
Religionslehre, 2 St. Im Sommerhalliahre: Christliche Glaubenslehre im Zusammenhange (Theologie und Anthropologie). Die Hauptbeweisstellen des N. T. wurden in der Ursprache gelesen und erläutert. Hr. Prof. Huth. Im Winterhalbjahre: Christologie des A. T. Hr. Prof. Frank.

Vathematik, 3 St. Arithmetik: Buchstabenrechnung. Dip Lehre von den Potenzen und Wurzeln, die Rechnungsarten mit Wurzelyrössen, Potenzen mit gebrochenen Exponenten, und imaginären Grössen. Sïmmtliche Lehren wurden durch Beispiele und Aufgaben erläutert und eingeülot.

Geometrie: Die Berechnung des Flärheninhalts ebener Figuren; die Lehre rom Kreise und der Aehnlichkeit der Figuren bis zum Schluss der Planimetrie. Die Lehrsätze und Aufgahen wurden in den Lehrstunden vollständig bewiesen und aufgelöst und dann von den Sthülern zur Uebung und zum bessern Verständnisse nach dazu gegebenen Figuren bearbeitet. Hr. Prof. Braun.

Physik, 2 St. Nach einer kurzen Repetition der Hauptsätze der Statik und Mechanik wurde die Lehre von den tropflbar - und elastisch-flüssigen Körpern vorgetragen. Sämmtliche Lehren wurden durch Fxperimente erläutert. Hr. Prof. B ra un.

Geschichte, 3 St. Griechische Geschichte und Geographie. Seit Weihnachten: Sächsische Geschichte (angefangen). Hr. Prof. A pel. Zeichnen, 2 St. Hr. Prof. Dietrich.

\section{Erste Classe (Selecta).}

Latein, 7 St. Davon I St. Fxtemporalien und Durchgehen der freien Ausarbeitungen.

I St. Disputirübungen. Es wurde über kleine schriftliche Ausarbeitungen disputirt, die Stellen aus lateinischen oder griechischen Autoren behandelten. Es opponirten jedesmal 2 Schüler, von denen der Eine auf das Formelle, der Andere auf das Materielle der vorliegenden Arbeit einzugehen pflegte.

5 St. Horat. Od. lib. II, 1-3. 6. 7. 10. 13. 14. 16. 17. 19. 20 Sat. lib. I, 9 10. lib. II, 1. Epist. lib. I, 7-12. Tacit. Agricola. Cursorisch: Plaut. trimmmus. Director.

Da der Director als Landtagsabgeordneter in der Zeit vom 21. Norember bis 17. December, während der landschaftlichen Sizungen, verhindert war, seine 4 Nachmittagsstunden zu ertheilen, so hatten die Herren Professoren Apel und Köhler die Gefälligkeit, dieselben zu übernehmen. Bei Hrn. Prof. $\Lambda$ pel wurden in 2 wöchent- 
lichen Vicariatstunden die Capitel 18-30 ron Cic. de off. lib. III, bei Hru. Prof. $\mathrm{K}$ öhler chenfalls in 2 wöchentlichen Stunden ausgewählte Oden von Horatius gelesen.

Griechisch, 6 St. Davon 1 St. Grammatik. Alle 14 Tage ein Extemporale.

3 St. Herod. lib. I. (cursorisch). Demosth. oratt. Chersones. and Pluil. III. Dire ctor.

2 St. Sophocl. Electra Homer. Nias lib. XVIII.-XXIV. Hr. Prof. Apetz.

Deutsch, 3 St. Im Sommerhalljahr: Geschichte, der deutschen Nationallitteratur bis Klopstock. Alle 4 Wochen ein Aufsatz. Declamationsübungen. Erklärung ausgewählter Klopstock'scher Oden. Uebungen im freien Vortrage. Hr. Prof Huth. Im Winterhallijahr: Ueber das deutsche Volksepos. Erklïrung Sthiller'scher Dramen. Freie Vorträge. Declamationen. Aufsätze. Hr. Prof. Frank.

Französisch, 2 St. Uebersetzung von Graf's Aufgaben zur Uebung des französischen Stils. I. Abth. S. 42-86. Gelesen wurde der Tartuffe von Molière und die Andromaque von Racine. Hr. Prof. Köhler.

Engliseh, 2 St. Formenlehre und Syntax nach Wagner's Schulgrammatik. Schriftliche und mündliche Uebungen. Gelesen wurde Goldsmith's Vicar of Wakefield Cap. 21-30. Hr. Prof. Köhler.

Hebräisch, 2 St. Die Syntax. Gelesen warde: I. Sam. c. 1-9. Exod. 1-14. Psalm. 12 - 15. 90-92. 96. Uehersetzung von Stellen aus dem uriechischen Texte des Matlhäus und Anderer in's Hebräische. Hr. Prof. Apetz.

Religionslehre, 2 St. Im Sommerhalbjahr: Kirehengeschichte bis Karl d. Gr. Hr. Prof. Huth. In Winterhallijahr: Kirchengeschichte von Karl d. Gr. bis zur Reformation. Hr. Prof. Frank.

Mathematik, 3 St. Arithmetik: Die Lehre von den Gleichungen mit einer und mehreren unbekannten Grössen; von den Logarithmen und logarithmischen Gleichungen; von den arithmetischen und geometrischen Reihen und deren Anwendungr. Sïmmtliche Leliren wurden durch Beispiele und Uehungsaufgaben erläutert und eingeübt. E be ne Trigon on etri e: Die trigonometrischen Functionen. Berechnung derselben aus gegebenen Stücken. Einrichtung und Gebrauch der trigonometrischen 'Tafeln. Die trigonometrische Auflösung der ebenen Dreiecke. Anwendung der Trigonometrie zur Auflösung astronomischer, geographischer and geometrischer Aufgaben. $\mathrm{Hr}$. Prof. Braun.

Physik, 2 St. Die Lehre von dem Lichte und den gebräuchlichsten optisphen Instrumenten durch Experimente und Zeichnungen erläutert 
und durch Auflösung entsprechender Aufgaben eingeübı. Hr. Prof. Braun.

Geschichte, 2 St. Neuere Geschichte von der Entdeckung Annerika's bis zum zweiten Pariser Frieden. Hr. Prof. A pel.

Alte Litteratur, 1 St. Geschichte der römischen Litteratur. Director. Zeichnen, 2 St. Hr. Prof. Dietrich.

Am Schluss des Schuljahres hat mir der Selectaner Julius Knipfer einen historisch-kritischen Versuch über die Lieder Ossian's als grössere freiwillige Arbeit übergeben.

\section{Frequenz.}

Die Zahl der Schüler betrug am Ende des vorigen Schuljahres 1556. Von diesen gingen ausser den 17 in der 46. Nachricht namentlich aufgeführten Abiturienten zu 0 stern v. J. alb :

aus Selecta:

18) Gustar Emil Steudemann aus Altenburg.

aus Prima:

19) Karl Friedrich Richard Voretzsch aus Altenbury.

aus 0 ber-Secunda:

20) Georg Ferdinand Bruno Reichardt aus Kauern.

21) Oskar Richard Kratzsch aus Schmölln.

22) Max Kanold aus Altenburg.

aus Mittel-Secunda.

23) Victor Edmund Dietz aus Altenburg.

24) Franz Arno Immanuel Henny aus Lucka.

25) Hermann Rudolph Wagner aus Altenburg.

26) 0 tt o Runkwitz aus Altenburg.

27) Theobald Robert Wille aus Altenburg.

In Laufe des Schuljahres gingen ab:

aus Prima:

18) Friedrich Wilhelm Ernst Foss aus Altenburg.

aus 0 ber-Secunda:

29) Karl O t to Daum aus Altenburg.

30) Karl Philipp Bernhard Steininer aus Altenlurg.

31) Franz Albert A ugust Schwepfinger aus Poniz.

ans Mittel-Secunda:

32) Franz Bernhard Gropp aus Altenburg. 
33) Balduin Ottomar Guido Kellberg aus Altenburg.

34) Thuisko Eduạd Ehrhard $t$ aus Altenburg.

Gestorben ist:

35) Bernhard Hempel aus Altenburg.

Dagegen wurden zn Ostern 20, zu Pfingsten 1, zusammen 21 Schiiler aufyenommen, mithin beträgt die Zahl der Schüler am Ende des Schuljahres 142, von denen sich 25 in Selecta, 32 in Prima, 30 in OberSecunda, 28 in Mittel-Secunda, 27 in Unter-Serunda befinden.

Das diesjährige schriftliche Maturitiits-Examen wurde in den Tagen vom 6. bis 9. Mä̀rz, das mündliche in den Vormittagsstunden des 23. und 24. März abgehalten. In Folge desselhen haben 11 Abiturienten ron dem Herzoglichen Consistorium in nachstehender Reihenfolgo die Erlaubniss zum A bgange zur Universität erhalten:

1) Schulze. 2) Knipfer. 3) Hannss. 4) Bräutigam. 5) (Schiffmann). 6) Reinhardt. 7) Scherf. 8) Reichardt. 9) Kirmse. 10) Geller. 11) (Sonntag von dem Gymnasium in Gera.) 


\section{Verze i c h n is s \\ de $r$}

\section{Schüler des Gymnasiums zu Ostern 1854}

n a o h der

durch das Weihnachtsexamen bestimmten Rangordnung,

nebst Angabe der Plätze, die sie durch das Johannis- und Michaelis-Examen erhalten habẹn.

( $t r$. bedeutet die zu Ostern 1853 Translocirten; $n_{\text {. }}$. die Neusufgenommenen; t bedeutet, dass ein Schüler Krankheitshalber oder aus einem andern triftigen Grunde das Examen nicht mitgemacht und in Folge davon seinen früheren Platz behalten hat.)

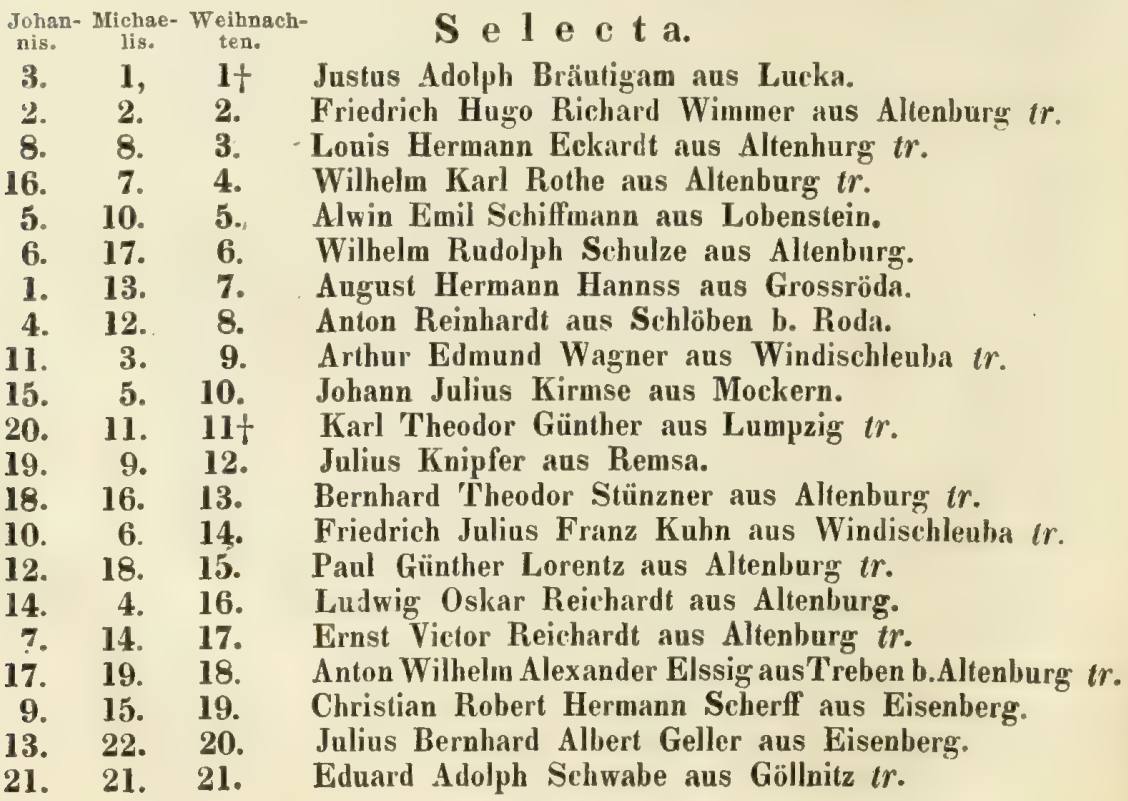


Johan- Michae- Weilnach-
nis. lis. ten.

23. 24. 22. Karl Gustav Eberhardt aus Roda $t r$.

22. 20. 23. Victor Eugen Huth aus Altenburg $t r$.

25. 25. 24. Traugott Hunnius aus Wolfersdorf $b$. Ronneburg $t r$.

24. 23. 25. Friedrich August Prüfer aus Hilbersdorf $t r$.

$$
\text { P } r \text { i m a. }
$$

8. 1. It Karl Heinrich Böttger aus Schmölln.

12. 3. 2. Friedrich Ernst Julius Taube aus Altenburg $t r$.

9. 7. 3. Richard Gustav Meissner aus Altenburg.

3. 10. 4. Karl Friedrich Paul Ludewig aus Eisenberg.

$2+\quad 6.5$. Arthur Hager aus Altenburg.

11. 9. 6. Richard Adolph Pietzsch aus Altenburg $\mathbf{~} r$.

19. 18. 7. Ernst Konon Löbe aus Rasephas.

1. 4. 8. Friedrich Konrad Wagner aus Altenburg.

21. 16. 9. Richard Pierer aus Altenburg.

20. 19. 10. Johannes Karl Friedrich Hesekiel aus Altenburg.

4. 5. 11. Julius August Robert Kirchhof aus Meuselwitz.

22. 14. 12. August Emil Busch aus Roda $t r$.

14. 13. 13. Leopold Bernhard Foss aus Altenburg $t r$.

23. 23. 14. Moritz Seidel aus Kahla n. $a$.

10. 15. 15. Johannes Felix Voretzsch aus Altenburg.

7. 12. 16. Alwin Robert Fischer aus Bocka.

17. 21. 17. Bernhard Heinrich Voretzsch aus Altenburg $t r$.

24. 24. 18. Otto Alexander Nebelsieck aus Wildenborn b. Zeitz $t r$.

6. 11. 19. Edmund Max Wagner aus Windischleuba.

18. 22. 20. Franz Julius Krause aus Altenburg $t r$.

5. 2. 21. Victor Ernst Emil Gustar Quaas ans Altenburg $t r$.

25. 30. 22. Ernst Gottlob Lehmann aus Altenburg $t r$.

15. 8. 23. August Hermann Oertel aus Tröbnitz.

13. 20. 24. Karl Gustar Osswald aus Altenburg $t r$.

16. 17. 25. Karl Gustar Pabst aus Altenburg.

26. 27. 26. Friedrich Julius Tenbner aus Ronneburg $t r$.

33. 32. 27. Fridolin Egmont Richard Bertuch aus Pölzig b. Ronneb. $(r$.

31. 29. 28. Theodor Kühn aus Wilchwitz $t r$.

32. 33. 29. Robert Theodor Buch aus Dobraschütz $t r$.

30. 26. 30. Karl Fritzsche aus Roda $t r$.

27. 25. 31. August Moritz Hermann Kretschmann aus Eisenberg $\boldsymbol{n}$. $a$.

28. 31. 32. Wilhelm Adolph Grässer aus Mosel.

$$
\text { O b e r - S e c u n d a. }
$$

1. 2. J. Julius Dähne aus Altenburg. 
Johan- Michae- Weihnach-
nis. lis.

3. 1. 2. Friedrich August Hermann Holzhauer ans Altenbury.

2. 14. 3. Reinhold Findeisen aus Kahla.

25. 3. 4. Karl Eduard Schürer aus Altenburg $t r$.

8. 4. 5. Karl Leopold Eduard Reichardt aus Altenburg $t r$.

6. 18. 6. Karl Friedrich Huth aus Altenburg.

5. 5. 7. Karl Theodor Weise aus Kosma.

4. 6. 8. Friedrich Leopold Theodor Földner aus Altenburg.

7. 17. 9. KarI Julius Pabst aus Altenburg

11. 7. 10. Ernst Otto Fritsche aus Altenburg $t r$.

239 11. Julius Theodor Engelmann aus Altenburg.

16. $16 \div$ 12. Rudolph Hempel aus Schmölln $n$. $a$.

21 11. 13. Karl Julius Bernhard Hinkel aus Berlin.

9. 15. 14. Karl Thilo Mïnzer aus Altenburg.

13. 19. 15. Robert Balduin Roth aus Mörsdorf.

12. 21. 16. Gustav Friedrich Jacohs aus Kahla.

10. 23. 17. Moritz Pierer aus Altenburg $t r$.

29. 10. 18. Rudolph Günther aus Ronneburg.

15. 12. 19. Arno Woldemar Ehrhardt aus Altenburg $i r$.

30. 13. 20. Julius Edaard Schultes aus Altenburg.

20. 20. 2I. Karl Eduard Ferdinand v. Mathy atus Altenburg $t r$.

18. 24. 22. Karl Albrecht Kunze aus Schmirchau $t r$.

14. 8. 23. Hugo Straube aus Bornshain.

22. 18. 24. August Klein aus Leipzig $t r$.

$1726.25 . \quad$ Vietor Edmund Dietz aus Altenburg $t r$.

19. 31. 26. Max Jsidor Meissner aus Altenburg $t r$.

31. 30. 27. Karl Victor Eduard Lange aus Altenburg.

33. 29. 28. Saladin Gleissner aus Rositz $t r$.

27. 22. 29. Johann Georg Volkmar Meinhardt a. Wintersdorf b. Lucka $t r$.

24. 32. 30. Karl Friedrich Hebenstreit aus Altenburg:

\section{M i t t e l-S e c u nda.}

1. 3. 1. Anton Rudolph Otto Kersten aus Altenburg.

9. 1. 2. Raimund Pierer aus Altenburg.

4. 6. 3. Johann Albert Paukert aus Wintersdorf b. Lucka.

11. 8. 4. Gustav Hofmann aus Altenburg $t r$.

6. 9. 5. Robert Ehrenfried Geyler aus Rochsburg b. Penig $n$. $a$.

7. 6. 6. Arno Lossius aus Zeutzsch b. Orlamïnda.

3. 5. 7. Kurt Wunder aus Altenburg.

5. 10. 8. Moritz Gustar Dietrich aus Altenburg:

8. 13. 9. Alexander Johann Pitzschel ans Menselwitz.

26. 18. 10. Albert Hugo Schlegel aus Regis $\boldsymbol{n}$. $a$. 
Johan- Michae- Weihnach-

24. 14. 11. Albert Lossius aus Zentzsch bei Orlamünda.

10. 7. 12. Otto Friedrich Ernst Steiniger aus Altenburg.

12. 12† 13. Adolph Reinhold Fritsche aus Altenburg.

17. 17. 14. Gustav Eduard Schneider aus Meuselwitz.

14. 22. 15. Martin Moritz Weber aus Rositz $n$. $a$.

16. 20. 16. Karl Friedrich Günther aus Altenburg $t r$.

18. 16. 17. Christian Emil Ritter aus Penig $t r$.

13. 11. 18. Ludwig Liuders aus Altenburg.

28. 21. 19. Karl Friedrich Oskar Hahn aus Thiemendorf $n$. $a$.

20. 24. 20. Eduard Julius Max Wille aus Altenburg.

21. 19. 21. Franz Robert Landmann aus Pölzig b. Ronneburg.

22. 23. 22. Constantin Friedrich Richard Löbe aus Altenburg $t r$.

15. 26. 23. Wilhelm Arno Jecke aus Ronneburg $n$. $a$.

23. 25. 24. August Wilhelm Adolph Meissner aus Penig $t r$.

19. 28. 25. Victor Lommer aus Altenburg $t r$.

25. 15. 26. Karl William Seidenfaden aus Altenburg $t r$.

29. 27. 27. Otto Mossdorf aus Altenburg $t r$.

27. 29. 28. Kuno Adelbert William Kellberg aus Altenburg $t r$.

\section{Un t e r - S e e u n d a.}

5. 5. 1. Valentin Kirmse aus Unterzetscha $n$. $a$.

1. 1. 2. Bernhard Hermann Musshacke aus Altenburg:

3. 3. 3. Ernst August Kühn-aus Altenburg.

7. 6. 4. Karl Friedrich Julius Dietzel aus Altenburg $n$. $a$.

4. 4. 5. Karl Robert Kirchhof aus Meuselwitz.

18. 9. 6. Richard Meinhardt aus Wintersdorf b. Lucka n. a.

14. 11. 7. Hugo Max Benndorf aus Langenleuba-Niederhain $n$. $a$.

9. 13. 8. Julius Gerth aus Fichtenhain $n$. $a$.

25. 12. 9. Hermann Louis Voigt aus Rositz $n, a$.

2. $2+10$. Theodor Julius August Mohrmann aus Altenbarg.

8. 8. 11. Karl Gustav Heinrich Hase aus Bobeck b. Eisenberg.

10. 14. 12. Max Joseph Ferdinand Geyer aus Treben b. Altenburg.

6. 7. 13. Ernst Felix Alphons Geyer aus Treben b. Altenburg.

11. 10. 14. Ernst Arthur Schädlich aus Altenburg.

13. 15. 15. Albert Otto Taube aus Altenburg.

12. 20. 16. Karl Georg Braune aus Altenburg.

22. 22. 17. Franz Rupertus Heinke aus Kosma. n. $a$.

17. 17† 18. Hugo Richard Geyer aus Treben b. Altenburg.

19. 19. 19. Friedrich Johannes Heinrich Lützelberger aus Altenburg.

23. 21. 20. Karl Leopold Heinrich Lützelberger aus Altenburg.

16. 16. 21. Oskar Heinrich Zetzsche aus Altenburg $n$. $a$. 
De colagpteris, quae 0scarus et Alfredus Brehm in Africa

Johan- Michae- Weihnach-

nis. lis. 18. 22. Ludwig Heinrich Zetzsehe aus Altenburg $n$. $a$.

24. 24. 23. Friedrich Karl Poppe aus Altenburg $n$. $a$.

20. 23. 24. Friedrich Theodor Hermann aus Altenburg $n$. $a$.

25. 26. 25. Kurt Oskar Mäckel aus Altenburg.

27. 25. 26. Gustav Emil Abt aus Altenburg $n$. a.

26. 27. 27. Rudolph Schuchmann aus Altenburg $n$. $a$. 